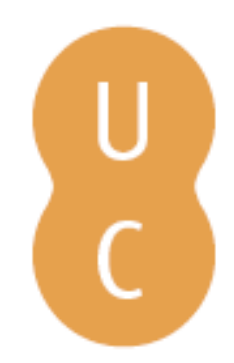

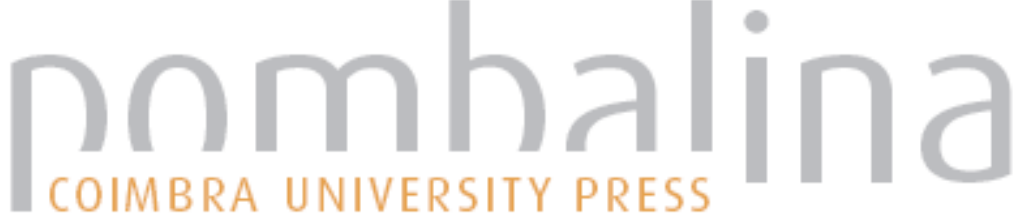

\section{Cultura organizacional, satisfação e cidadania organizacional}
Autor(es):
Neves, José; Lopes, Albino
Publicado por: Imprensa da Universidade de Coimbra
URL persistente:
URI:http://hdl.handle.net/10316.2/32744
DOI:
DOI:http://dx.doi.org/10.14195/978-989-26-0452-7_2

Accessed : $\quad$ 26-Apr-2023 10:57:42

A navegação consulta e descarregamento dos títulos inseridos nas Bibliotecas Digitais UC Digitalis, UC Pombalina e UC Impactum, pressupõem a aceitação plena e sem reservas dos Termos e Condições de Uso destas Bibliotecas Digitais, disponíveis em https://digitalis.uc.pt/pt-pt/termos.

Conforme exposto nos referidos Termos e Condições de Uso, o descarregamento de títulos de acesso restrito requer uma licença válida de autorização devendo o utilizador aceder ao(s) documento(s) a partir de um endereço de IP da instituição detentora da supramencionada licença.

Ao utilizador é apenas permitido o descarregamento para uso pessoal, pelo que o emprego do(s) título(s) descarregado(s) para outro fim, designadamente comercial, carece de autorização do respetivo autor ou editor da obra.

Na medida em que todas as obras da UC Digitalis se encontram protegidas pelo Código do Direito de Autor e Direitos Conexos e demais legislação aplicável, toda a cópia, parcial ou total, deste documento, nos casos em que é legalmente admitida, deverá conter ou fazer-se acompanhar por este aviso.

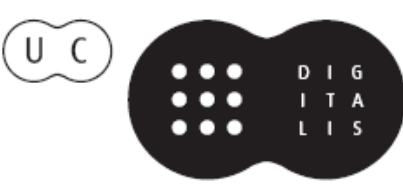




\section{A. DUARTE GOMES - ANTÓNIO CAETANO JOSÉ KEATING • MIGUEL PINA E CUNHA

\author{
Coordenadores
}

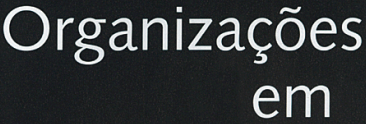

Transição

Contributo da Psicologia do Trabalho

e das Organizaçōes 
(Página deixada propositadamente em branco) 


\section{A. DUARTE GOMES - JOSÉ KEATING ANTÓNIO CAETANO - MIGUEL PINA E CUNHA Coordenadores}

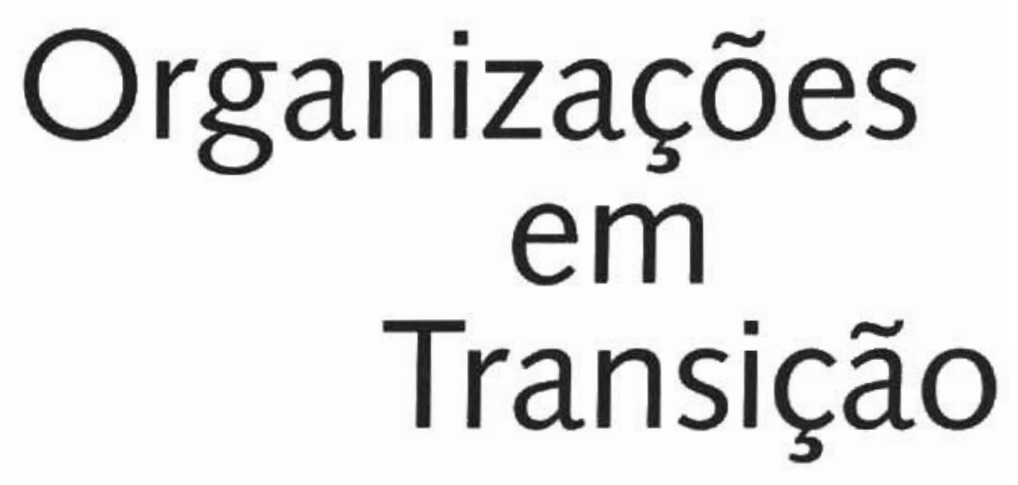

Contributos da Psicologia do Trabalho e das Organizações 


\section{COORDENACÁO EDITORIAL \\ Imprensa da Universidade de Coimbra \\ CONCEPÇÃO GRAFACA \\ António Barros \\ EXECUÇÃO GRAFICA \\ G.C. - Gráfica de Coimbra, Lda. \\ Palheira - Assafarge - Apart. 3068 \\ 3001-453 Coimbra Codex}

ISBN

972-98225-3-0

DEPOSITO LEGAL.

153435/00

(c) JunHo 2000. Imprensa da Universidade de Coimbra 


\title{
CULTURA ORGANIZACIONAL, SATISFAÇÃO E CIDADANIA ORGANIZACIONAL
}

\author{
Jose Neves e Albino Lopes \\ INSTITUTO SUPERIOR DE CIEANCIAS DO TRABALHO E DA EMPRESA (ISCTE)
}

I. INTRODUÇÃO

Presentemente, a pressão da produtividade e da qualidade constitui uma realidade que caracteriza as instituições públicas. Esta pressão é acentuada pelo incremento dos comportamentos cívicos que sensibilizam cada cidadão para os seus direitos enquanto utente dos serviços públicos e pressiona as pessoas que prestam o serviço público a desenvolverem mais esforços no sentido da interacção e da cooperação. Constituem evidência desta tendência, quer o programa de modemização da administração pública, quer os programas de qualidade habitualmente designados por programa dos zero defeitos.

A modernização da Administração Pública, constitui actualmente uma preocupação central em todos os países desenvolvidos. De facto, numerosos autores (Gore, 1994; Silveira e Trindade, 1992; Albi et al., 1997; Defélix, 1997 e Correia, 1997), afirmam que se assiste hoje, à mais profunda crise de confiança, a nível mundial, na Administração Pública, motivada pelos sucessivos défices orçamentais, derivados da ineficácia financeira dos serviços administrativos e, por um défice de desempenho, que leva os utentes do serviço público a sentirem dificuldades no seu relacionamento com tal serviço.

Neste contexto, e na opinião de Pettigrew (1997), a maior parte dos países desenvolvidos foram forçados a alterar o seu modelo organiza- 
tivo. não só em termos de estruturas administrativas, mas também, de processos de trabalho e do comportamento dos funcionários. Para tal promoveram a desburocratização de procedimentos, com vista à maior transparência dos actos administrativos e promoção da descentralização das instâncias de decisão, com o reforço da autonomia de gestão e dos poderes reguladores, por parte das estruturas regionais e locais (autarquias e serviços públicos).

O nosso país não foge à regra. Um relatório oficial, de 1994, relativo à situação da Administração Pública portuguesa, sustentava que esta faz das regras e procedimentos um fim em vez de um meio, denotando um especial desprezo pelo cumprimento de metas e objectivos, bem como pelos cidadãos e suas necessidades. $O$ esforço que desde há cerca de 20 anos vem sendo desenvolvido no sentido da modernização administrativa, está longe de ter atingido os objectivos inicialmente propostos, como é reconhecido no diploma legal que cria o Sistema de Qualidade em Serviços Públicos (D. L n ${ }^{\circ}$ 166-A/99 de 13 de Maio). Apesar de alguns sucessos pontuais, o diploma reconhece que os custos económicos e sociais, resultantes da ausência de qualidade dos serviços públicos, são cada vez maiores e mais pesados para o cidadão e para os agentes económicos. A nossa conviç̧ão é a de que não é sustentável manter, por muito mais tempo, a existência de uma administração pública paralizante, formalista, desmotivada e muito pouco preocupada com a qualidade do serviço prestado, fazendo do utente um administrado e não um cliente.

Nas dificuldades constatadas pelos responsáveis citados acerca da modernização administrativa, conjugam-se duas dimensões igualmente importantes: a garantia da qualidade dos serviços prestados e o funcionamento interno das organizações. É esta segunda dimensão do problema que constitui a base da reflexão que se segue, cuja ênfase é posta na actuação dos próprios funcionários.

Uma observação atenta das frequentes disfuncionalidades que no dia-a-dia da Administração Pública se manifestam ao nível da pouca qualidade do serviço, do mau atendimento ao utente, da lentidão do serviço, do desperdício de tempo, de material e de recursos humanos e no gosto pela rotina, induz a pensar se não estamos perante um conjunto de valores estruturantes destas disfuncionalidades. Se sim, o desafio para qualquer programa de intervenção organizacional, consiste em conseguir uma mudança de cultura 
organizacional, passível de orientar a acção de cada pessoa e das respectivas unidades, de uma forma mais eficiente. Daqui resulta que o espaço organizacional se torna mais complexo e por vezes paradoxal em termos do seu funcionamento. Por vezes, as decisões de natureza organizacional conflituam porque se confrontam com dilemas entre o curto e o longo prazo, entre o desenvolver ou aperfeiçoar um produto, entre o custo e o benefício, ou entre 0 inovar e o assegurar a estabilidade do "status quo", a ponto de autores como Cameron (1986), tratarem o problema da eficácia organizacional como um paradoxo, no sentido de esta se definir na base de contrastes e dilemas.

Este fenómeno acentua a importância de se compreenderem os aspectos culturais de um contexto organizacional específico, tal como a crença no seu estatuto de condicionadores da excelência organizacional. Quer a satisfação, quer as estratégias individuais de actuação organizacional, recebem assim uma base maior de compreensibilidade, e emerge uma maior explicitação de um modelo de intervenção organizacional.

A cultura organizacional é um conceito que aparece referido de um modo mais sistemático na literatura organizacional a partir dos finais da década de 70, geralmente associado a percepções avaliativas, e estruturado na base de múltiplas dimensões. A literatura organizacional revela a cultura organizacional como um conceito multidimensional, assumindo diferentes significados para diferentes pessoas, emergindo por isso problemas relacionados com a definição do conceito. A este propósito, Ott (1989) refere 73 palavras ou frases usadas para definir cultura organizacional. Apesar desta falta de acordo, a definição de Schein (1990) «Organizational culture can be defined as a set of core values, behavioural norms, artefacts and behavioural patterns which govern the way people in an organization interact with each other and invest energy in their jobs and in the organization at large», reúne algum consenso entre os investigadores, e proporciona uma base conceptual válida de operacionalização e de avaliação empírica. Apesar da variedade de processos que se encontram na origem da obtenção dos dados, e na impossibilidade de se definirem dimensões universais da cultura organizacional, alguns autores recorrem ao modelo das tipologias como um meio de contornar o problema da multidimensionalidade, argumentando que considerar a cultura como um conjunto integrado, uma totalidade, é uma forma mais adequada de abordar o problema, do que fazê-lo na perspectiva das dimensōes. Quinn e colaborado- 
res, apoiados em três dimensões do funcionamento das organizações (controle/flexibilidade, interno/externo, e meios/fins), sugerem uma tipologia de cultura organizacional, baseada em quatro quadrantes: apoio, inovação. objectivos e regras.

Em relação aos métodos de avaliação, os investigadores dividem-se em termos de opinião, entre as metodologias qualitativas e quantitativas. Mas apesar da falta de acordo, pensamos como Zammuto e Krakower (1989), para quem os dados de natureza quantitativa constituem apenas um ponto de partida para investigar a cultura, os quais deverão ser complementados e validados com dados oriundos de metodologias qualitativas, como entrevistas, sessões de "feedback" , análise documental, etc., produzindo-se assim uma medida mais consistente e mais aprofundada da cultura.

A satisfação organizacional é um conceito com grande longevidade em termos de produção científica. $\mathrm{Na}$ base desta longevidade, está a crença no importante papel das atitudes individuais e das relações interpessoais, como determinantes da produtividade.

Emergem assim aspectos da esfera individual e social, traduziveis em atitudes e emoções, ou seja em respostas afectivas de satisfação ou insatisfação no trabalho, com um peso importante na definição das condições físicas e ambientais do trabalho, bem como na formulação dos processos de gestão (tipo de chefia, grau de formalização do trabalho, tomada de decisões, estilo de comunicação, fluxo da informação, etc.), e da concepção e organização do trabalho. Por isso se investiga o impacto da liderança na satisfação dos liderados, a importância da variedade, flexibilidade, finalidade, autonomia, etc., no posto de trabalho que permite ao seu detentor estados afectivos intrinsecamente gratificantes (crescimento, responsabilidade, informação, etc.), e extrinsecamente produtivos (mais quantidade e mais qualidade de trabalho, menos "stress", menos absentismo e rotação). Trata-se de uma consequência da descoberta de que «além de braço, o homem é igualmente coração».

A satisfação no trabalho traduz uma resposta emocional e afectiva, gratificante, que resulta da situação de trabalho. E quais e quantas as facetas do trabalho com impacto na satisfação? Apesar de o trabalho se afigurar multifacetado, e de tais facetas assumirem pesos diferentes, a análise da literatura revela como principais e comuns as seguintes: trabalho desafiante, equidade na recompensa, condições físicas e ambientais de trabalho e relacionamento interpessoal. 
Por trabalho desafiante entende-se, a variedade, autonomia e "feedback" que o posto de trabalho tem, que possibilite ao trabalhador o uso pleno das suas capacidades físicas e mentais e fazer um trabalho estimulante. $O$ carácter desafiante possui alguns limites; quando em excesso, o resultado é de frustração e de sentimentos de incapacidade.

A equidade na recompensa traduz a necessidade de os sistemas e politicas de promoção, de remuneração e de outros beneficios reflectirem justiça, clareza e transparência e serem capazes de responder às expectativas do trabalhador. Mais que o valor absoluto de cada um destes aspectos, é o seu valor relativo que mais contribui para gerar respostas de satisfação ou de insatisfação.

As condições físicas e ambientais de trabalho dizem respeito não só às condições gerais de temperatura, luminosidade, higiene, como às mais específicas (equipamentos e ferramentas de trabalho, condições de segurança, localização e inserção da organização no meio local, etc.).

O relacionamento interpessoal diz respeito às relações formais e informais que se estabelecem numa situação de trabalho e, que acontecem entre colegas, superiores e subordinados, possibilitadoras da construção de uma auto-identidade, de relações de cooperação e ajuda e de amizade. Os estudos são unânimes em destacar a importância da relação chefe-subordinado para a satisfação no trabalho. Esta é aumentada quando o chefe é compreensivo e amigo, se interessa pelos colaboradores, escuta as suas opiniões e os elogia na sequência de um bom desempenho. Neste estudo, as dimensões de satisfação reveladas, após análise factorial, foram: objectivos e gestão global, recompensas, relacionamento com a chefia directa, relacionamento com colegas, relacionamento com a direcção do Departamento e características do trabalho.

Algumas medidas de satisfação têm sido desenvolvidas, umas mais relacionadas com a satisfação em geral, outras mais dirigidas a aspectos específicos da satisfação. Grande parte dos estudos recorre a questionários estruturados para fornecerem uma medida directa de satisfação, outros porém usam isoladamente ou em conjugação entrevistas aos sujeitos, inferindo daí o grau de satisfação dos mesmos. $\mathrm{Na}$ base de tais opções estão diferentes modelos teóricos e diversas perspectivas metodológicas. Entre nós, existe um estudo com uma amostra portuguesa (Lima, Vala e Monteiro, 1989), que procura operacionalizar um conjunto de modelos considerados relevantes para o estudo da satisfação. Os resultados obtidos revelam a grande complexidade que é a satisfação organizacional. 
Uma situação que igualmente contribui para a satisfação no trabalho é o grau de adequação das estratégias de actuação organizacional. Tradicionalmente, são as taxas de rotação e de absentismo que são utilizadas como indicadores do estado de insatisfação. Elevado absentismo ou uma alta taxa de rotação de pessoas, seriam o equivalente de baixa satisfação. Contudo, numerosos estudos empíricos (Mobley, 1977, Porter e Steers, 1973, Staw, 1980) dão conta da fraca relação entre estas variáveis, o que significa a existência de outras variáveis a mediarem a relação entre satisfação e absentismo ou rotação. Na sequência dos trabalhos de Farrell, (1993), Rusbult et al., (1988) e Caetano e Vala, (1995), os quais preconizam uma síntese integradora de diferentes modalidades de resposta organizacional, disponibilizadas pelos sujeitos de uma organização no seu contexto de trabalho, analisámos as estratégias de actuação organizacional dos funcionários de uma instituição autárquica portuguesa e explorámos igualmente alguns dos determinantes de tais opçōes comportamentais.

Saída externa, voz, lealdade, negligência (Rusbult et al., 1982) e saída interna (Caetano e Vala, 1995), constituem os padrōes tipificadores de estratégias de actuação organizacional que os sujeitos adoptam, não só como resposta à insatisfação, mas também como meio de concretizarem os objectivos pessoais no espaço organizacional com os recursos de poder de que dispõem. Por saída externa entende-se um comportamento activo de alguém que abandona a organização, donde se desvincula psicologica e contratualmente. Por voz entende-se um comportamento igualmente activo, caracterizado pela assertividade do sujeito na discussão dos problemas da organização. A lealdade traduz um comportamento relativamente passivo face ao dia a dia da organização, caracterizado pela atitude de assistir aos acontecimentos. A negligência traduz uma diminuição do esforço e do empenhamento na organização, ou seja uma quase ausência real ou simbólica da organização. A saída interna traduz um comportamento activo de mudança de local de trabalho mas dentro da mesma organização.

Como definido anteriormente na apresentação e contextualização do problema, Caetano e Vala (1995), sugerem no final do seu trabalho sobre estratégias de actuação organizacional, que este tipo de estudos deveria considerar, entre outras variáveis, a relação entre estas estratégias e a cultura organizacional. 


\section{O MODELO TEÓRICO DOS VALORES CONTRASTANTES E O MODELO DE ANALISE}

O modelo dos valores contrastantes tem origem no modelo de eficácia desenvolvido por Quinn e Rohrbaugh (1981;1983), e parte de três dimensões organizacionais bipolares e relacionadas entre si. $O$ modelo pode representar-se por dois eixos, à volta dos quais três dimensões se distribuem e formam quatro quadrantes. Tal como está referido na figura I, a primeira dimensão está representada no eixo horizontal, sendo a extremidade esquerda o posicionamento da orientação interna e o extremo direito o posicionamento da orientação externa. Do ponto de vista da orientação interna, a ênfase consiste em desenvolver os recursos humanos e manter estável e cooperante o ambiente de trabalho. Do ponto de vista externo, o objectivo organizacional é desempenhar as actividades para crescer e adquirir recursos.

A segunda dimensão está representada no eixo vertical e varia entre a flexibilidade (topo do eixo) e o controle (base do eixo). Neste par de valores contrastantes o controle enfatiza a necessidade de hierarquia, e controle na cooperação entre funções, enquanto a flexibilidade acentua a importância da iniciativa individual, da rapidez e da adaptabilidade organizacional.

A terceira dimensão meios/fins traduz o dilema permanente que está subjacente aos diversos modelos organizativos, a saber: crescimento e aquisição de recursos através da inovação e rapidez de resposta em oposição à estabilidade e controle através do sistema de informação e de comunicação. Subjacente a esta dimensão e implícitos em cada quadrante, estão os modelos teóricos do funcionamento organizacionais. Os modelos são os seguintes: o modelo dos objectivos racionais em que o fundamental é o alcance dos objectivos definidos, o modelo do sistema aberto no qual a principal finalidade é a adaptação, o modelo das relaçōes humanas que enfatiza a motivação das pessoas e a descentralização das decisões e o modelo dos processos internos que preconiza como principal finalidade a integração.

Esta dimensão reflecte um contínuo de meios-fins, o que traduz os conflitos inerentes aos problemas da prioridade (planeamento versus rapidez de resposta), da importância (valor dos recursos humanos versus produtividade) e do tempo ( estabilidade e controle para garantir segurança e qualidade versus flexibilidade e rapidez para assegurar crescimento e competitividade).

A integração destas orientações origina quatro quadrantes culturais com as seguintes designações: cultura de regras, cuja orientação é para a estabili- 
dade e para o interno e que se caracteriza pelo acentuar da importância dos sistemas implícitos (valores e crenças partilhados) e explícitos (regras e regulamentos) de controle; cultura de apoio, cuja orientação é para a flexibilidade e para o interno e que se caracteriza pelo sentido de pertença e de responsabilidade criados por elevados níveis de envolvimento e de participação; cultura de inovação, cuja orientação é para o externo e para a flexibilidade e que se caracteriza pela capacidade de tomar medidas adequadas para fazer face às pressōes externas e de flexibilizar os comportamentos e processos de funcionamento interno; a cultura de objectivos, cuja orientação é para o externo e para a estabilidade e que se caracteriza por enfatizar a importância do significado, clareza e direcção, que os papeis individuais e institucionais e os objectivos internos e externos, devem evidenciar.

No conjunto, a cultura de inovação e a cultura de objectivos, dirigem-se à dinâmica externa da organização e enfatizam a capacidade da organização para a mudança e flexibilidade, enquanto a cultura de apoio e a cultura da regras se dirigem à dinâmica interna, e se orientam para a segurança e estabilidade. $O$ funcionamento organizacional é assim perspectivado na base de orientações culturais contrastantes.

Cada um destes tipos de cultura constitui um conjunto de pressupostos básicos, que estão subjacentes aos processos de motivação, de liderança, de decisão, de eficácia, etc.. Os valores, as crenças, as atitudes e os pressupostos fundamentais que são partilhados pelos membros de uma organização constituem a essência da cultura organizacional. Tal partilha traduz uma visão comum acerca de diversos aspectos da natureza e do funcionamento organizacional, como por exemplo: relacionamento interpessoal e intergrupal, objectivos, regras e normas da organização, comportamentos apropriados, modos de resolver problemas, etc.. De acordo com os tipos de cultura anteriormente referidos, é de esperar que existam diferenças na visão comum, entre os diferentes tipos culturais. Numa cultura de inovação, são as crenças na importância e valor da criatividade, da ino-

42 vação, da flexibilidade, da receptividade à mudança, etc., o objecto de partilha comum entre os membros. Numa cultura de objectivos, são os valores da produtividade, da eficiência, da maximização de resultados, etc., que constituem a base dominante. Numa cultura de apoio, é partilhada a crença na importância da cooperação, do espírito de grupo, do bem estar das pessoas, etc.. Numa cultura de regras, a base para a comunidade de crenças, centraliza-se nos valores da ordem, da hierarquia, dos sistemas de informação, do controle, etc.. 
Que vantagens traz conceber a cultura organizacional na base do modelo dos valores contrastantes? Pensamos que as mesmas se podem resumir ao seguinte: integração dos vários tipos de cultura organizacional nas principais teorias organizacionais, formando a justaposição de valores, uma estrutura teórica integrada que possibilita um entendimento mais profundo e balanceado da cultura; clarificação do conteúdo conceptual mediante uma representação gráfica do mesmo, contribuindo para resolver o problema de sobreposições, ambiguidades e indefinições de conteúdos do conceito de cultura; salvaguarda da característica fundamental dos sistemas humanos e organizacionais, que é o dinamismo, ao contextualizá-lo a partir da sua forma de funcionamento paradoxal e contrastante; permitir a formulação e investigação de hipóteses de estudo, a partir da forma como cada tipo de cultura se relaciona com outros critérios comparativos, como a estratégia, o desempenho, os recursos humanos, a liderança, etc., desenvolvendo e operacionalizando planos de investigação capazes de possibilitarem comparações entre diversos estudos, sem que o conceito de cultura se altere; dar conta do carácter de multiplicidade e de simultaneidade, das várias orientações contrastantes prosseguidas por cada organização no seu funcionamento.

Modelo das relaçōes humanas Flexibilidade Modelo dos sistemas abertos

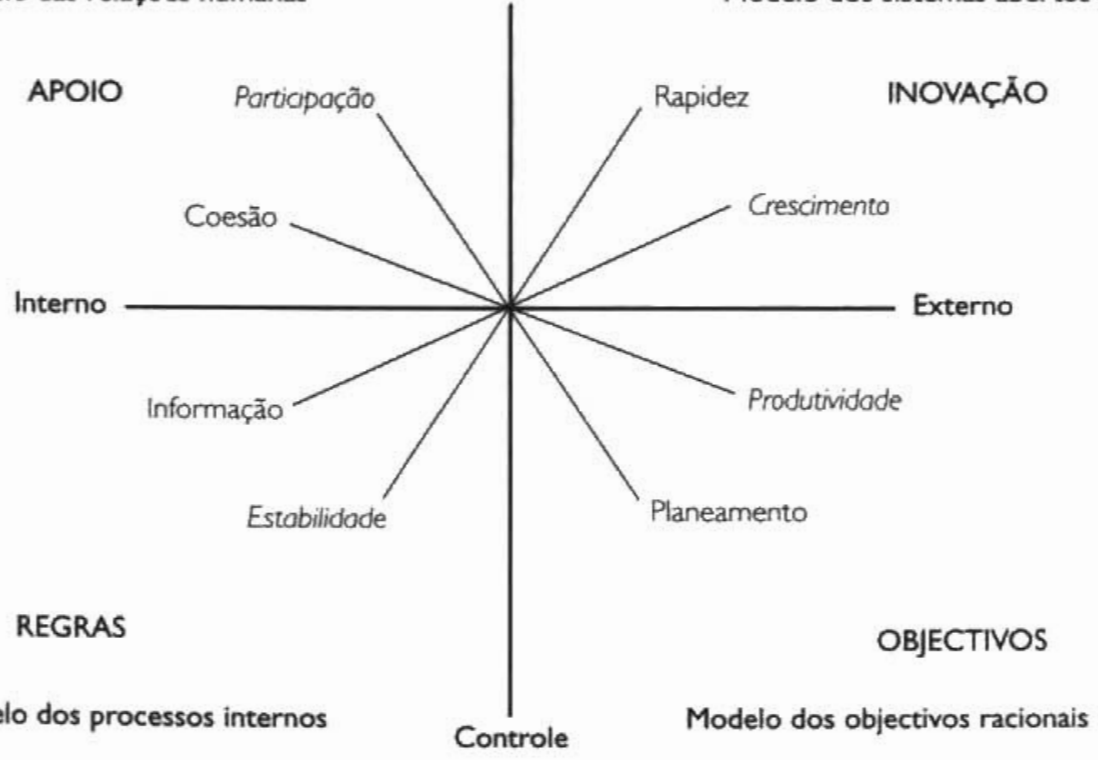

FGURA I - Modelo dos valores contrastantes de Quinn e colaboradores 
A utilização deste modelo para estudar a cultura organizacional assume que as organizações se caracterizam por traços ou dimensões comuns e por isso se torna necessário adoptar critérios metodológicos que possibilitem análises comparativas. Esta postura retoma a discussão sobre os métodos mais apropriados para avaliar a cultura. De um lado da barreira estão os defensores das metodologias qualitativas e mais de cariz antropológico, como sendo as mais apropriadas para avaliarem a componente essencial da cultura (valores e pressupostos fundamentais); do outro lado estão os defensores dos métodos quantitativos com o argumento de que os mesmos possibilitam a comparação dos resultados, quer em termos do nível de análise (individual, grupal e organizacional), quer em termos de tempo (passado, presente e futuro), quer em termos do real e actual versus ideal ou desejável, quer ainda em termos de relação com outras variáveis organizacionais, como a estratégia, a liderança, a gestão de conflitos, a tomada de decisão, a gestão de recursos humanos, etc.

Mais do que explorar a não conciliação de tais diferenças, é consensual entre numerosos investigadores, que é mais proveitoso uma análise cuidada das vantagens e inconvenientes de cada modalidade de abordagem no estudo da cultura e uma combinação dos métodos mais apropriados para avaliar o conteúdo da cultura em causa. Hofstede et al (1990), Denison e Spreitzer (1991), Siehl e Martin (1982), Zammuto e Krakower (1991), Yeung et al (199I), Quinn et al (|981), são alguns dos autores que desenvolveram estudos empíricos, na linha dos que defendem a vantagem da complementaridade das várias metodologias de abordagem. Um caminho que será igualmente percorrido no estudo empírico inserido neste trabalho e que adiante se apresenta.

O modelo de análise que definimos (ver figura seguinte) integra três tipos de variáveis independentes: a cultura organizacional, a satisfação organizacional e a eficácia percebida das opções comportamentais. Pressupomos que a cultura organizacional pode incentivar a adopção de uma ou outra 44 opção comportamental, como sendo a mais apropriada do ponto de vista da organização. Por exemplo, numa cultura de regras, a adopção de estratégias comportamentais de voz poderão ser não incentivadas pela organização, independentemente de existirem ou não razões de insatisfação. A ser verdade este pressuposto, as opções comportamentais terão na sua determinação outros factores que não os exclusivamente intraindividuais. Igualmente admitimos que as respostas afectivas em relação ao trabalho e que se expressam 
sob a forma de um índice de satisfação, predisporão de forma diferente os sujeitos para fazerem uso das diversas opções comportamentais. Neste pressuposto, pessoas satisfeitas tenderão a fazer menos uso de estratégias comportamentais como a saída ou a negligência, do que pessoas insatisfeitas. A terceira variável que utilizamos no nosso modelo diz respeito ao ganho percebido por parte do sujeito decorrente da opção comportamental que adoptou. Neste pressuposto, será de esperar que quanto maior tiver sido o ganho percebido em relação à opção de comportamento utilizada, maior será a probabilidade de tal opção vir a ser utilizada.

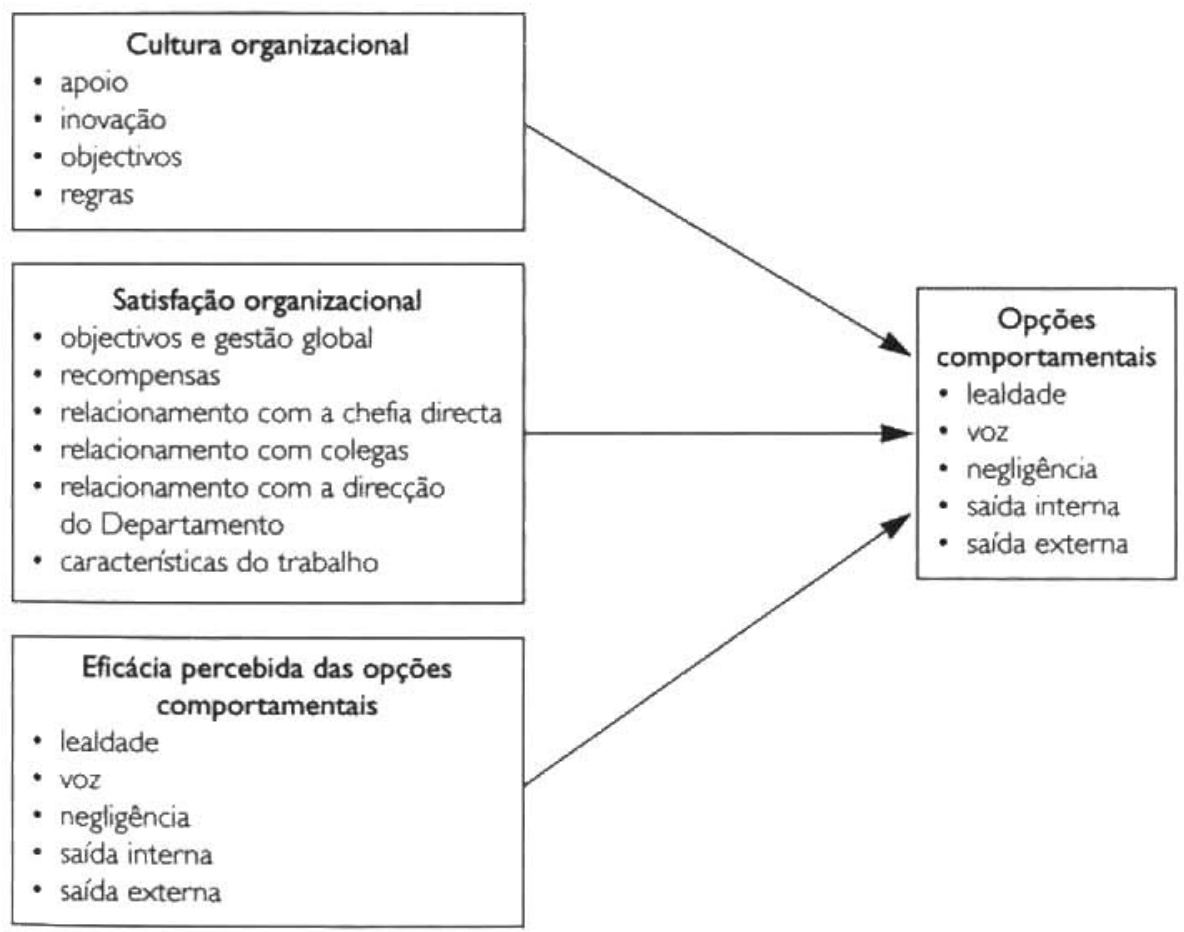

FIGURA 2 - Modelo de análise

3. MÉTODO

Este trabalho fez uso dos seguintes métodos:

- Realização de entrevistas individualmente e em grupo, com vista a clarificar aspectos da cultura e do funcionamento geral da instituição; 
- Recolha de informação dos funcionários da instituição, com base nas respostas obtidas a um questionário sobre cultura organizacional, satisfação e opções comportamentais;

- Análise estatística dos dados quantitativos:

- Análise documental (balanço social, relatórios de actividade e outra informação julgada relevante).

\section{I. ENTREVISTAS}

Com a finalidade de compreender a orgânica e funcionamento da instituição, bem como eventuais problemas existentes, foram efectuadas entrevistas individualmente e em grupo, cuja duração variou entre 60 e 90 minutos.

A população entrevistada totalizou 32 pessoas e incluiu elementos com funções de chefia ou de relevância técnica integrados em diferentes niveis hierárquicos da organização.

Para a definição da população a entrevistar, utilizou-se o critério do posicionamento hierárquico e/ou da relevância técnica em termos de função.

As entrevistas apesar do seu carácter semiestruturado, seguiram um guião do qual se destacavam dois objectivos fundamentais: obter a cooperação do entrevistado e colher informação relevante para a percepção do funcionamento da instituição. Observou-se durante todo este processo uma excelente colaboração dos entrevistados.

\subsection{O QUESTIONARIO}

Dada a especificidade dos objectivos deste estudo, numa primeira etapa foi necessário construir o questionário, o qual, após uma aplicação piloto, foi

46 finalizado e utilizado como instrumento de recolha de dados quantitativos.

\subsubsection{CONSTRUÇĀO DO QUESTIONÁRIO}

O questionário utilizado foi preparado com base no material informativo recolhido das entrevistas e no modelo de análise referido anteriormente. 
É composto de 2 partes. A primeira diz respeito à cultura e consiste nas questões do modelo FOCUS 93, com 35 questões que referenciam normas, valores e pressupostos básicos, igualmente relacionados com as vertentes interna e externa da organização. $O$ questionário contém perguntas distribuídas pelas quatro orientações do modelo dos valores contrastantes e são avaliadas numa escala unidimensional tipo Likert. $A$ amplitude da resposta varia entre seis posições qualitativas, com o seguinte significado: I. nunca/ninguém; 2 . poucos/raramente; 3 . alguns/por vezes; 4 . muitos/com frequência; 5 . quase todos/quase sempre e 6. todos/sempre. As razões do uso da escala de Likert prendem-se com a possibilidade de tornar possivel medidas independentes de cada quadrante e com a possibilidade de captar mais realisticamente os quadrantes e respectivas relações. Trata-se de um instrumento de avaliação da cultura organizacional, baseado no modelo dos valores contrastantes de Quinn e colaboradores e desenvolvido por um grupo de universitários de 13 paises, dos quais um dos responsáveis por este estudo é o representante por parte de Portugal. A segunda parte integra um conjunto de questões preparadas na sequência das entrevistas e inventariadas com base nos estudos de Lima et al. (1989) e de Caetano et al. (1995). As mesmas avaliam aspectos ligados à satisfação no trabalho (objectivos e gestão global, recompensas, relacionamento com a chefia directa, relacionamento com os colegas, relacionamento com a direcção do departamento e características do trabalho), às estratégias de actuação organizacional (lealdade, voz, negligência, saída externa e saída interna) que as pessoas adoptam face ao seu grau de satisfação e à eficácia das estratégias de actuação organizacional.Todas as questões estão estruturadas em termos de resposta, numa escala tipo Likert.

\subsubsection{ESTUDO PILOTO E APLICAÇĀO DO QUESTIONÁRIO}

Após o envio de uma nota informativa a todos os trabalhadores da instituição pela Presidência da instituição, na qual se dava informação sobre o estudo em curso e se fazia um convite para a participação de todos no mesmo, foi feita uma aplicação piloto a 27 elementos da instituição.

Feitas as análises e correcções necessárias decorrentes desta aplicação piloto, o questionário foi finalizado e aplicado aos trabalhadores da instituição. 
A aplicação foi efectuada nas instalações da instituição, quer individualmente, quer em pequenos grupos e assistida por responsáveis por este estudo e outros elementos preparados para o efeito. Cada respondente gastou em média 20 minutos.

O total dos questionários respondidos foi de 272 , ou seja, $63 \%$ do total dos efectivos da instituição. Os dados quantitativos e respectivas análises estatísticas foram efectuadas com recurso ao programa SPSS (Statistical Package for the Social Sciences), um software estatístico desenvolvido para uso nas Ciências Sociais.

\section{AMOSTRA E RESULTADOS}

\section{I CARACTERIZAÇĀO DA AMOSTRA}

O questionário foi respondido por 272 pessoas, o que representa $63 \%$ do universo dos colaboradores da instituição.

No quadro I apresenta-se a distribuição dos inquiridos pelas variáveis de caracterização sociodemográfica e de inserção organizacional.

Destaque para o facto de cerca de $53 \%$ da amostra ter uma idade superior a 40 anos e uma escolaridade reduzida. Destaque igualmente para o facto $58 \%$ ter uma antiguidade na instituição superior a 11 anos.

\subsection{OS RESULTADOS}

\subsection{CULTURA ORGANIZACIONAL.}

Para o agrupamento das variáveis em tipos de cultura utilizou-se o critério teórico-empírico, com base nos resultados do grupo FOCUS. Para efeito de análise e interpretação dos dados agrupou-se a estrutura da instituição em cinco conjuntos na base das afinidades funcionais. Os agrupamentos são: DAF (Departamento Administrativo-Financeiro), DAU (Departamento de Administração Urbanística), DSGU (Departamento de Serviços Gerais e Urbanos), DEAS (Divisão de Educação e Acção Social), DC (Divisão Cultural) e DJ (Divisão Jurídica) e OUTROS. O quadro seguinte resume os resultados 
QUADRO I

CARACTERIZAÇĀO DA AMOSTRA

\begin{tabular}{|c|c|c|}
\hline & $(n=272)$ & $\%$ \\
\hline $\begin{array}{l}\text { SEXO } \\
\text { M } \\
\text { F } \\
\text { Sem resposta }\end{array}$ & $\begin{array}{r}188 \\
66 \\
18\end{array}$ & $\begin{array}{r}69,1 \\
24,3 \\
6,6\end{array}$ \\
\hline $\begin{array}{l}\text { Idade } \\
<20 \\
21 \text { a } 30 \\
31 \text { a } 40 \\
41 \text { a } 50 \\
51 \text { a } 60 \\
>60 \\
\text { Sem resposta }\end{array}$ & $\begin{array}{r}2 \\
31 \\
87 \\
100 \\
36 \\
8 \\
8\end{array}$ & $\begin{array}{r}0,7 \\
11,4 \\
32,0 \\
36,8 \\
13,3 \\
2,9 \\
2,9\end{array}$ \\
\hline $\begin{array}{l}\text { Habilitações } \\
4^{a} \text { classe } \\
\text { até ao } 6^{\circ} \\
\text { até ao } 9^{\circ} \\
\text { até ao } 12^{\circ} \\
\text { bacharelato } \\
\text { licenciatura } \\
\text { outro } \\
\text { Sem resposta }\end{array}$ & $\begin{array}{r}113 \\
30 \\
29 \\
42 \\
11 \\
29 \\
9 \\
9\end{array}$ & $\begin{array}{r}41,5 \\
11.0 \\
10,7 \\
15,4 \\
4,0 \\
10,7 \\
3,3 \\
3,3\end{array}$ \\
\hline $\begin{array}{l}\text { Estado civil } \\
\text { Solteiro } \\
\text { Casado } \\
\text { Outro } \\
\text { Sem resposta }\end{array}$ & $\begin{array}{r}31 \\
206 \\
13 \\
22\end{array}$ & $\begin{array}{r}11,4 \\
75,7 \\
4,8 \\
8,1\end{array}$ \\
\hline $\begin{array}{l}\text { Antiguidade } \\
<3 \text { anos } \\
3 \text { a } 5 \text { anos } \\
6 \text { a } 10 \text { anos } \\
11 \text { a } 15 \text { anos } \\
16 \text { a } 20 \text { anos } \\
>20 \text { anos } \\
\text { Sem resposta }\end{array}$ & $\begin{array}{r}46 \\
20 \\
41 \\
40 \\
62 \\
56 \\
7\end{array}$ & $\begin{array}{r}16,9 \\
7,3 \\
15,1 \\
14,7 \\
22,8 \\
20,6 \\
2,6\end{array}$ \\
\hline
\end{tabular}




\begin{tabular}{|l|r|r|}
\hline Nivel hierárquico & 28 & 10,3 \\
Chefia & 217 & 79.8 \\
Não chefia & 27 & 9,9 \\
Sem resposta & & \\
\hline Tipo de Chefia & 12 & 42,9 \\
Encarregado & 3 & 10,7 \\
Chefe de Secção & 3 & 10,7 \\
Chefe de Repartição & 7 & 25,0 \\
Chefe de Divisão & 0 & 0,0 \\
Director de Departamento & 3 & 10,7 \\
Sem resposta & & \\
\hline Departamento & 21 & 7,7 \\
DAF & 50 & 18,4 \\
DAU & 99 & 36,4 \\
DSGU & 24 & 8,9 \\
DEAS, DC e DJ & 45 & 16,5 \\
Outros sectores & 33 & 12,1 \\
Sem resposta & & \\
\hline
\end{tabular}

(média e desvio padrão) por cada tipo de cultura, na instituição global e nos diversos agrupamentos. Igualmente se incluem os dados relativos à fidelidade de cada tipo de cultura, bem como o índice de significância da diferença de médias entre agrupamentos.

QUADRO 2

RESULTADOS DA CULTURA ORGANIZACIONAL

\begin{tabular}{|c|c|c|c|c|c|c|c|c|c|c|c|}
\hline & Cultura & $\begin{array}{c}\mathrm{N}^{\circ} \\
\text { de } \\
\text { itens }\end{array}$ & $\mathrm{m}$ & $\begin{array}{l}C M \\
d p\end{array}$ & $\alpha$ & $\begin{array}{c}\text { DAF (1) } \\
m \quad d p\end{array}$ & $\begin{array}{c}\text { DAU (2) } \\
m \quad d p\end{array}$ & $\begin{array}{cc}\text { DSGU (3) } \\
m \quad d p\end{array}$ & $\begin{array}{c}\text { DEAS (4) } \\
m \text { dp }\end{array}$ & $\begin{array}{c}\text { Outros (5) } \\
m \quad d p\end{array}$ & P \\
\hline \multirow{4}{*}{50} & Apoio & 10 & 3.02 & .90 & .87 & $2.73 \quad .59$ & $2.87 \quad .83$ & $3.24 \quad 89$ & 2.66 .73 & $3.14 \quad 1.07$ & $.011^{*}$ \\
\hline & Inovaçẫo & 7 & 3.02 & .74 & .64 & $3.01 \quad .71$ & $2.87 \quad .67$ & 3.19 .78 & 2.77 .59 & $3.01 \quad .76$ & ns \\
\hline & Objectivos & 8 & 3.18 & .90 & .83 & $3.18 \quad .58$ & $2.76 \quad .67$ & 3.47 .86 & $\begin{array}{|ll|}2.73 & .87\end{array}$ & \begin{tabular}{|ll}
.17 & .95
\end{tabular} & $.000^{*}$ \\
\hline & Regras & 10 & 2.99 & .77 & .79 & $3.28 \quad .52$ & $2.76 \quad .70$ & $3.11 \quad .84$ & $2.87 \quad .87$ & $3.01 \quad .63$ & ns \\
\hline
\end{tabular}

Escala: | - de modo nenhum... 6-muitissimo

* Pares diferenciados: 
Os resultados aqui apresentados reflectem a forma como as pessoas percepcionam tais realidades. Cada um dos quadrantes, exprime a tensão da orientação respectiva, configurando-se a totalidade do conjunto como a expressão actual das tensões da cultura na CM. Os resultados indicam que em termos da cultura, as percepções são semelhantes e relativamente homogéneas. Em termos de tensão, observam-se valores médios relativamente semeIhantes em todos os tipos de cultura, o que revela um equilibrio global dos vários tipos de cultura. Contudo, a cultura de objectivos é aquela que revela uma percepção média mais elevada. Entre os vários agrupamentos, as diferenças observadas em relação aos perfis, apesar de não muito acentuadas, revelam-se significativamente diferentes em relação à cultura de apoio e de objectivos, sendo as percepçōes do DSGU as mais elevadas e significativamente diferentes em termos estatísticos das percepções dos restantes agrupamentos. Esta ênfase nos objectivos poderá ser explicada pelo actual acentuar da pressão política no sentido de a administração pública, onde se inclui o poder autárquico, se balizar por critérios de produtividade, ancorando no plano e no orçamento os pilares principais de todo o funcionamento autárquico.

Para além dos perfis da cultura da instituição como um todo, e em que se constata ser a cultura de objectivos aquela que é percepcionada mais intensamente, podemos observar também os perfis revelados ao nível dos diferentes departamentos. Como se constata, existem diferenças em termos de orientações dominantes entre os vários departamentos, quer do ponto de vista da intensidade perceptiva (médias mais baixas versus médias mais elevadas), quer do ponto de vista das ênfases dominantes (controle no DAF, flexibilidade no DAU, competitividade no DSGU, eixo inovação-regras no DEAS, $D C$ e DJ e eixo apoio-objectivos nos OUTROS)

\subsubsection{SATISFAÇĀO NO TRABALHO}

Os dados descritivos dos resultados agregados em função de seis dimensões obtidas através de análise factorial em componentes principais da escala de satisfação composta por 32 questões, são apresentados no quadro seguinte. 
QUADRO 3

MÉDIAS E PERCENTAGENS DAS DIVERSAS DIMENSÖES DA SATISFAÇÃO

\begin{tabular}{|l|c|c|c|c|c|c|c|c|c|l|}
\hline Factores & $\begin{array}{c}\mathrm{N}^{\circ} \\
\text { de } \\
\text { itens }\end{array}$ & alfa & $\mathbf{m}$ & $\mathrm{dp}$ & $\begin{array}{c}\text { Daf } \\
\mathbf{m} \\
(\mathbf{1})\end{array}$ & $\begin{array}{c}\text { Dau } \\
\mathbf{m} \\
(\mathbf{2})\end{array}$ & $\begin{array}{c}\text { Dsgu } \\
\mathbf{m} \\
(3)\end{array}$ & $\begin{array}{c}\text { Deas } \\
\mathbf{m} \\
(4)\end{array}$ & $\begin{array}{c}\text { Out } \\
\mathbf{m} \\
(5)\end{array}$ & $\mathrm{P}$ \\
\hline Objectivos e gestāo global & 7 & .90 & 2.8 & .80 & 2.8 & 2.5 & 3.0 & 2.3 & 2.7 & $.001 *$ \\
\hline Recompensas & 5 & .73 & 2.1 & .68 & 2.1 & 1.8 & 2.4 & 1.8 & 1.9 & $.000 *$ \\
\hline Relacionamento com chefia directa & 3 & .85 & 3.3 & 1.01 & 3.3 & 3.0 & 3.5 & 3.3 & 3.1 & $.050^{*}$ \\
\hline Relacionamento com colegas & 3 & .85 & 3.2 & .98 & 3.1 & 3.2 & 3.3 & 3.3 & 3.1 & $\mathrm{~ns}$ \\
\hline Relacionamento com director & 3 & .84 & 3.0 & .95 & 3.2 & 3.1 & 3.2 & 2.8 & 2.7 & $.011^{*}$ \\
\hline Características do trabalho & 4 & .85 & 3.4 & .87 & 3.5 & 3.2 & 3.6 & 3.2 & 3.2 & $.032 *$ \\
\hline
\end{tabular}

Escala: 1 - nada satisfeito ... 5-muitissimo satisfeito

- Pares diferenciados:

"Objectivos e gestāo global" (3/4, 3/2, 3/5)

"Recompensas" $(3 / 4,3 / 2,3 / 5,3 / 1)$

"Relacionamento com chefia directa" (3/2)

"Relacionamento com direç̧ão do departamento" (2/5, 3/5)

"Caracteristicas do trabalho" $(3 / 5,3 / 2)$

No global, são as recompensas e os objectivos e gestão global, os factores em que a satisfação é mais baixa; o trabalho realizado, o relacionamento com as chefias directas e com os colegas constituem os factores que revelam um mais elevado grau de satisfação. De realçar ainda a boa consistência interna dos factores e a diferença significativa entre os vários agrupamentos em relação aos vários factores da satisfação.

\subsubsection{OPÇŌES COMPORTAMENTAIS E EFICACIA PERCEBIDA}

A forma como os indivíduos reagem no seu quotidiano, aos aspectos

52 negativos que percepcionam nas organizações, ou como programam a sua actuação pessoal, pode ser tipificada de acordo com as seguintes estratégias comportamentais:

Voz - Desempenhar um papel activo, de forma individual ou colectiva, na resolução dos problemas de funcionamento organizacional.

Lealdade - Estratégia passiva, que consiste em esperar que as situações evoluam positivamente. 
Negligência - Estratégia reactiva, que consiste em reduzir o empenhamento organizacional ao mínimo, sem correr riscos de puniçāo.

Saída Extema - Estratégia activa, que consiste em programar, ou mesmo anunciar, a saída da organização.

Saída Intema - Comportamento activo, que consiste em manisfestar o desejo de mudar de departamento.

Em termos da teoria das organizações, considera-se que a opção de Voz ou de acção afirmativa é aquela que mais facilita a mudança e a inovação organizacional. A Lealdade é a estratégia que facilita o equilíbrio organizacional, tendo consequências positivas quando existe uma estratégia organizacional clara e aceite. As estratégias de Saída, por sua vez, embora possam envolver custos organizacionais elevados, podem ter efeitos positivos no desbloqueamento de rotinas disfuncionais. Finalmente, a Negligência é claramente avaliada como uma modalidade de acção organizacionalmente negativa.

No quadro seguinte apresentam-se os resultados relativamente a cada uma das opçōes comportamentais averiguadas.

\section{QUADRO 4}

MÉDIAS DAS OPÇOEES COMPORTAMENTAIS

\begin{tabular}{|l|c|c|c|c|c|c|c|c|l|}
\hline $\begin{array}{l}\text { Opçōes } \\
\text { comportamentais }\end{array}$ & alfa & M & DP & $\begin{array}{c}\text { Daf } \\
\text { M } \\
\text { (1) }\end{array}$ & $\begin{array}{c}\text { Dau } \\
\mathbf{M} \\
(2)\end{array}$ & $\begin{array}{c}\text { Dsgu } \\
\mathbf{M} \\
(3)\end{array}$ & $\begin{array}{c}\text { Deas } \\
\mathbf{M} \\
(4)\end{array}$ & $\begin{array}{c}\text { Outros } \\
\mathbf{M} \\
(5)\end{array}$ & $P$ \\
\hline Lealdade & .52 & 2.9 & .99 & 2.7 & 2.6 & 3.3 & 2.9 & 3.0 & $.011^{*}$ \\
\hline Voz & .52 & 2.7 & 1.02 & 2.6 & 2.8 & 2.7 & 2.9 & 2.7 & $\mathrm{~ns}$ \\
\hline Negligência & .40 & 2.2 & .96 & 1.9 & 2.4 & 2.3 & 1.9 & 2.3 & $\mathrm{~ns}$ \\
\hline Saida externa & .85 & 1.8 & 1.05 & 2.1 & 1.8 & 1.6 & 1.9 & 2.0 & $\mathrm{~ns}$ \\
\hline Saida interna & .89 & 2.0 & 1.24 & 1.6 & 2.0 & 2.1 & 1.4 & 2.4 & $.018^{*}$ \\
\hline
\end{tabular}

Escala: 1 - de modo nenhum ... 5-inteiramente

- Pares diferenciados:

"Saida Interna" $(5 / 4,5 / 1)$

Como se pode observar, Lealdade e Voz são as estratégias de actuação mais disponíveis e a Saída Externa a menos saliente. A cada uma destas opções comportamentais podem ser associados beneficios, no sentido de eficácia esperada destas actuaçōes na vida profissional (V. Quadro 5). 
Em geral sobressai a eficácia da Lealdade, da Voz e da Negligência. As opções pela saída em qualquer das modalidades revela eficácia reduzida.

QUADRO 5

MÉDIAS DA EFICÁCIA DAS OPÇŌES COMPORTAMENTAIS

\begin{tabular}{|l|c|c|c|c|c|c|c|c|}
\hline $\begin{array}{l}\text { Opçōes } \\
\text { comportamentais }\end{array}$ & M & DP & Daf & $\begin{array}{c}\text { Dau } \\
(1) \\
\mathbf{m}\end{array}$ & $\begin{array}{c}\text { Dsgu } \\
(3) \\
\mathbf{m}\end{array}$ & $\begin{array}{c}\text { Deas } \\
(\mathbf{4}) \\
\mathbf{m}\end{array}$ & $\begin{array}{c}\text { Outros } \\
\mathbf{s}(5) \\
\mathbf{m}\end{array}$ & $\mathrm{P}$ \\
\hline Lealdade & 2.9 & 1.00 & 2.5 & 2.8 & 3.2 & 2.7 & 3.0 & $\mathrm{~ns}$ \\
\hline Voz & 2.8 & 1.04 & 2.6 & 2.6 & 3.0 & 2.8 & 2.7 & $\mathrm{~ns}$ \\
\hline Negligência & 2.7 & 1.16 & 2.7 & 2.6 & 2.9 & 2.1 & 2.7 & $\mathrm{~ns}$ \\
\hline Saída externa & 2.6 & 1.22 & 2.4 & 2.2 & 2.7 & 2.5 & 2.9 & $\mathrm{~ns}$ \\
\hline Saída interna & 2.2 & 1.05 & 1.7 & 2.0 & 2.3 & 1.7 & 2.8 & $.000 *$ \\
\hline
\end{tabular}

Escala: 1 - discordo completamente ... 5-concordo completamente

- Pares diferenciados:

"Saida Interna" (5/4, 5/1, 5/2, 5/3, 3/4, 3/1)

\subsection{DETERMINANTES DAS OPČ̄ES COMPORTAMENTAIS}

Pretende-se agora averiguar os determinantes que influenciam a activação de cada uma das opções comportamentais descritas anteriormente. Para isso, realizaram-se análises de regressão múltipla para cada opção comportamental, considerando como variáveis independentes as dimensões da satisfação, as

54 dimensões de cultura e a eficácia percebida das opções comportamentais. Os resultados das análises de regressão múltipla apresentam-se no quadro seguinte. Para cada variável dependente é apresentada a variabilidade (R2) explicada por cada um dos conjuntos de variáveis independentes, o grau de significância de cada medida de variabilidade (estatística $F$ e nível de significância p) e a importância relativa de cada variável independente (coeficientes beta estandardizados) para a explicação de cada uma das variáveis dependentes. 
QUADRO 6

DETERMINANTES DAS OPÇÕES COMPORTAMENTAIS

\begin{tabular}{|c|c|c|c|c|c|}
\hline Variáveis & voz & lealdade & negligência & $\begin{array}{c}\text { saída } \\
\text { externa }\end{array}$ & $\begin{array}{c}\text { saida } \\
\text { interna }\end{array}$ \\
\hline $\begin{array}{l}\text { Cultura organizacional } \\
\text { Apoio } \\
\text { Inovação } \\
\text { Objectivos } \\
\text { Regras } \\
\end{array}$ & .21 & $\begin{array}{l}.21 \\
.29\end{array}$ & & -.27 & \\
\hline$R^{2}$ & .04 & .22 & & .07 & \\
\hline$F$ & 8.01 & 23.83 & & 13.57 & \\
\hline$p$ & .005 & .000 & & .000 & \\
\hline $\begin{array}{l}\text { Satisfação organizacional } \\
\text { Relacionamento com colegas } \\
\text { Relac. com. Direcção do Depart. } \\
\text { Relac. com a chefia directa } \\
\text { Objectivos e gestão global } \\
\text { Características do trabalho } \\
\text { Satisfação com a recompensa } \\
\text { Satisfação global }\end{array}$ & .28 & $\begin{array}{l}.20 \\
.21\end{array}$ & 2.1 & -.13 & $\begin{array}{l}-.23 \\
-.17\end{array}$ \\
\hline$R^{2}$ & .08 & .12 & .05 & .10 & .12 \\
\hline$F$ & 14.95 & 11.96 & 8.23 & 18.39 & 12.35 \\
\hline$p$ & .000 & .000 & .004 & .000 & .000 \\
\hline $\begin{array}{l}\text { Eficácia das opçōes } \\
\text { comportamentais } \\
\text { Voz } \\
\text { Lealdade } \\
\text { Negligência } \\
\text { Saída externa } \\
\text { Saída interna }\end{array}$ & .23 & $\begin{array}{l}.16 \\
.19\end{array}$ & $\begin{array}{r}-.18 \\
.26 \\
.28\end{array}$ & $\begin{array}{r}-.14 \\
.45\end{array}$ & $\begin{array}{r}-.22 \\
.51\end{array}$ \\
\hline$R^{2}$ & .08 & .07 & .21 & .21 & .30 \\
\hline $\mathrm{F}$ & 8.92 & 7.71 & 17.45 & 26.78 & 43.71 \\
\hline $\mathrm{P}$ & .000 & .000 & .000 & .000 & .000 \\
\hline
\end{tabular}


Observando os resultados inseridos no quadro anterior, constata-se a partir do conjunto das variáveis independentes consideradas, ser a percepção de eficácia das opções comportamentais a variável mais explicativa das opções comportamentais, seguida pela satisfação. A cultura organizacional contribui pouco para explicar as diversas opções comportamentais. Assim, de um modo mais analítico, a cultura organizacional contribui para explicar principalmente da opção pela lealdade (22\%), ou seja, quanto maior a orientação para a competitividade e para o exterior da organização por parte da Câmara, maior a propensão para a adopção de comportamentos de lealdade.

Considerando agora cada uma das orientações de cultura em termos da sua influência sobre cada uma das opçōes comportamentais, constata-se que a cultura de inovação influencia as opções da lealdade e da voz. A orientação apoio influencia embora em sentido inverso a opção saída externa. A opção lealdade é também influenciada pela cultura de objectivos.

Entre satisfação e opções comportamentais, a variância das opções lealdade e saída interna são explicadas em $12 \%$, e as restantes opções comportamentais são explicadas na sua variabilidade em percentagens inferiores. Observando agora a influência de cada uma das dimensões da satisfação sobre as opçōes comportamentais, verifica-se que a dimensão relacionamento com a chefia directa influencia as opções da voz e da lealdade, a influência em sentido inverso das dimensōes relacionamento com o director e características do trabalho sobre a opção saída interna, a influência de sentido inverso sobre a saída externa da satisfação global e a influência do relacionamento com o director sobre a negligência. A voz revela influência da dimensão relacionamento com a chefia directa. Apenas a lealdade e a saída interna se revelam pluri-influenciadas.

Relativamente à eficácia das opções comportamentais, a sua influência sobre as opções comportamentais é maior do que a observada com a cultura e com a satisfação. Assim, com excepção das opções voz e lealdade,

56 todas as restantes opções são explicadas na sua variabilidade em mais de $21 \%$. A percepção de eficácia da saída interna revela uma influência sobre as opções da negligência e da saída interna. A percepção de eficácia da opção negligência revela influenciar os comportamentos de negligência, de lealdade $e$ de voz. Por sua vez a percepção de eficácia da voz influencia as opções da voz e da lealdade e, em sentido inverso, as opções da negligência e da saída interna. Tal como se observou já em outras situações, também aqui cada uma 
das opções comportamentais se revela pluri-influenciada. Resumindo e fazendo uma leitura mais impressiva, dir-se-á que o modelo se revela bastante adequado, na medida em que a variância explicada pelas diversas variáveis independentes, atinge em alguns dos casos, níveis considerados relevantes para este tipo de estudos. Igualmente se poderá concluir que a ordem de grandeza pela qual as diferentes opções comportamentais são determinadas, é representada em $1^{\circ}$ lugar pela eficácia percebida das mesmas, depois pela satisfação, e por fim, pela cultura organizacional, o que significa um maior peso na determinação das opções comportamentais, por parte dos factores intrapessoais, do que factores externos ao sujeito.

\section{DISCUSSÃO E CONCLUSŌES}

Tratando-se de um diagnóstico organizacional com o intuito de melhor conhecer o funcionamento da instituição e de potencializar os aspectos facilitadores da implementação de um programa de melhoria da qualidade, uma leitura dos resultados possibilita algumas constatações de relevância para os objectivos em curso. Assim, pode afirmar-se que a cultura da instituição se revela facilitadora da implementação da qualidade de serviços, uma vez que a tendência dominante é a cultura de objectivos e esta se alicerça em dois pilares importantes de qualquer programa de melhoria da qualidade: a produtividade e a preocupação com o utente. Esta ambiência facilitadora é acompanhada da situação em matéria de satisfação, a qual é positiva em relação ao relacionamento pessoal e hierárquico e à valência intrínseca do trabalho realizado. As opções comportamentais percebidas como mais eficazes são as activas e de identificação com a instituição. Lealdade e voz são mesmo as estratégias comportamentais mais disponíveis.

Em termos teóricos, e apesar das limitações da amostra, algumas conclusões se podem extrair a respeito dos conceitos em análise e sua ligações, e dos processos de medida utilizados.

Acerca do conceito de cultura organizacional, a estrutura tipológica representada pelo modelo dos valores contrastantes, revela-se consistente e descriminativa, o que comprova idênticos resultados por nós obtidos em outros estudos (Neves, 1996). Já no que diz respeito à sua capacidade de influenciar as opções comportamentais por parte dos indivíduos, o seu contri- 
buto não se revelou muito importante, o que sugere que do ponto de vista teórico, são mais importantes os aspectos emocionais e a ideia de mudança ou sequência de opções comportamentais (o sujeito mudar de opção ao sabor da eficácia da mesma), do que a influência exercida pela cultura de uma organização. Sobre a satisfação, também aqui os resultados revelam a consistência habitual de resultados, quer em termos de dimensões, quer de capacidade discriminativa. Relativamente às opções comportamentais, os resultados sugerem a possibilidade de se pensar a relação entre satisfação e opções comportamentais numa amplitude maior de opções do que as tradicionalmente associadas ao absentismo. Esta diversidade de opções de comportamento é ainda apoiada pelo facto de cada uma ser determinada de forma diversa por dimensões também diversas.

Outro aspecto que do ponto de vista teórico merece ser considerado diz respeito às variáveis explicativas das opções comportamentais dos sujeitos. Neste estudo são as variáveis intraindividuais, como os estados afectivos inerentes à satisfação ou a perceção de eficácia obtida das opções que maior influência exercem ao nível das opções comportamentais. Factores extra individuais como a cultura, evidenciaram fraca relação com as opçōes de comportamento que cada sujeito adopta no seu contexto de trabalho. Factores relacionais e intergrupais ou comportamentos políticos poderão igualmente ser factores fortemente preditores das opções comportamentais. Assim, comportamentos de grupo ou recursos de poder poderão constituir talvez importantes factores mediadores das opções comportamentais.

Do ponto de vista prático, os resultados permitem validar o uso destes conceitos no diagnóstico e intervenção organizacional. Contudo, ao nível da operacionalização da variável dependente, importa ter presente a forma diversificada que cada uma das opções comportamentais pode assumir, consoante o nível de análise é o individual ou o grupal. Igualmente importa considerar, em cada um dos níveis de análise, a influência que os recursos de poder existentes

58 permitem na adopção de cada uma das estratégias ou opções comportamentais, quer estas sejam entendidas de uma forma estática, quer de um modo sequencial (hoje a pessoa faz uma opção, mas amanhã faz outra). 


\section{REFERENCIAS}

ALBI E., PÁRAMO, J. M., \& CASASNOVAS, G. L (1997). Gestión pública- Fundamentos, técnicas e cosos. Barcelona: Editorial Ariel, AS.

CAETANO, A e VALA, J. (1995). Estratégias de actuação organizacional. InVala, J., Monteiro, M.B. Lima, L e Caetano, A. Psicologia social das organizações, Celta Editora, Oeiras

CAMERON, K. S. (1986). Effectiveness as paradox consensus and conflict in conceptions of organizational effectiveness. Manogement Science, vol. 32, n5, May, 539-553.

Conselho Económico e Social (1999, 21 de Junho e 12 de Julho). Plano Nacional de Desenvolvimento Económico e Social- PENEDES.

CORREIA, D. (1997). Resistência à mudança e estratégias de superação: A situação de um municipio. Porto: Ediçōes Universidade Fernando Pessoa.

Decreto-Lei n. 166 do DRgr. N. ${ }^{\circ}$ । I I-AV99, de 13 de Maio.

DEFÉLIX, C. (1997). Entreprises de service public: Une seule gestion des ressources humaines? Reveue Française de Gestion, $n^{\circ}$ | I5, Septembre-Octobre, p., I30-140.

DENISON, D. R e SPREITZER. G. M. (1991). Organizational culture and organizational development a competing values approach. In R Woodman e W. A. Pasmore, (eds.). Research in Organization Change and Development, Vol 5, 1-21. Greenwich, Conn:JAl Press.

FARRELL, D. (1983). Exit, voice, loyalty, and neglect as responses to job satisfaction: a multidimensional scaling study. Academy of Manogement joumal, 26, 569-607.

GORE, AL, JR (1994). Reinventar a administração pública: Da burocracia à eficácia. Lisboa: Quetzal Editores.

HOFSTEDE, G., NEUIJEN, B., OHAY, D. D. e SANDERS, G. (1990). Measuring Organizational Cultures: A Qualitative and Quantitative Study across Twenty Cases. Administrative Science Quarterly, 35, 286-316.

LIMA, L. VALA, J. e MONTEIRO, M. B.(1989). Os determinantes da satisfação organizacional: confronto de modelos. Análise Psicológica, IV (3-4), 414-457.

MOBLEY, W. H. (1977). Intermediate linkages in the relationship between job satisfation and employee turnover. Joumal of Applied Psychology, 62: 237-240.

NEVES. J. G. (1996). Clima Organizacional, Cultura Organizacional e Gestão de Recursos Humanos: Portugal no contexto de outros países. Tese de Doutoramento, ISCTE, Lisboa.

OTT. J. S. (1989). The organizational culture perspective, Dorey Press, Chicago.

PETTIGREW, A. (1997) Le new public management conduit à un nouveau modèle hybride public-privé. Reveue Française de Gestion, $n^{\circ}$ 1 15, Septembre-Octobre, p., 113-120.

PORTER, LW. e STEERS, R.M. (1973). Organizational, work, and personal factors in employee turnover and absenteeism. Psychological Bulletin, 80: 151-176.

QUINN, R. E. e CAMERON. K. S. (1983)., Organizational Life Cycles and Shifting Criteria of Effectiveness: some preliminary evidence., Management Science, vol. 29 (1). 33-51.

QUINN, R. E. e ROHRBAUGH. J., (1981). A competing values approach to Organizational Effectiveness. Public Productivity Review, June. 122-140.

RUSBULT, C. E., FARREL, D., ROGERS, G e MAINOUS III, A. G. (1988). Impact of exchange variables on exit, voice, loyalty, and neglect: an integrative model of responses to declining job satisfaction. Academy of Management Review, 31 (3): 599-627.

SHEIN. E.H., (1990). Organizational culture. American Psychologist, vol. 45, n² 2, 109-1 19. 
SIEHL, C. e MARTIN. J. (1988) Measuring organizational culture: mixing qualitative and quantitative methods. In M. O. Jones, M. D. Moore e Snyder (Eds.), Inside Organizations: understanding the human dimension. 79-103. Newbury Park. CA: Sage.

SILVEIRA, P., \& TRINDADE, N. (1992). A gestão da administração pública: Usos e costumes, manios e anomalias ( ${ }^{*}$ ed.). Lisboa: Editorial Presença.

STAW, M. B. (1980). The consequences of toumover. Joumal of Occupational Behovior, I: 253-273.

VALA. J.. MONTEIRO, M.B., LIMA. L. e CAETANO, A. (1995). Psicologia social das organizações, Celta editora, Oeiras

YEUNG, A., BROCKBANK, W., e ULRICH, D. (1989). Organizational culture and human resource practices: an empirical assessment. In R. Woodman e W. A. Pasmore. (Eds.). Research in Organization Change and Development. Vol 5. 59-82. Greenwich. Conn: JAI Press.

ZAMMUTO, R. F. e KRAKOWER, J.Y. (1989). Quantitative and qualitative studies of organizational culture. In R. Woodman e W. A. Pasmore, (Eds.). Research in Organization Change and Development, Vol 5, 83-1 14, Greenwich, Conn: JAl Press.

O projecto Focus é uma iniciativa de um grupo de investigadores de diferentes paises, para desenvolver um instrumento internacional de avaliaçāo da cultura organizacional, baseado no modelo dos valores contrastantes. Os investigadores, bem como as respectivas cidades e paises são:

\section{Nome}

Gaston De Cock e Karel DeWitte

Claude Lemoine

Nancy Papalexandris e Dimitri Bourantas

Imre Branyiczki

Enzo Spaltro, Gincarlo Barnaber e Fábio Carati

Paul Koopman e Jaap van Muijen

Jorge Correia Jesuíno e José Gonçalves das Neves

Horia Pitariu

José-Maria Peiró e Vicente Gonzales-Roma

Michael West

Zoran Susanj

Eduard Konrad e Michael Kline

DavidTurnipseed

$\begin{array}{ll}\text { Cidade } & \text { País } \\ \text { Lovaina } & \text { Bélgica } \\ \text { Rouen } & \text { França } \\ \text { Atenas } & \text { Grécia } \\ \text { Budapeste } & \text { Hungria } \\ \text { Bolonha } & \text { Itália } \\ \text { Amesterdão } & \text { Holanda } \\ \text { Lisboa } & \text { Portugal } \\ \text { Cluy } & \text { Roménia } \\ \text { Valência } & \text { Espanha } \\ \text { Sheffield } & \text { Inglaterra } \\ \text { Rijeka } & \text { Croácia } \\ \text { Ljubljana } & \text { Eslovénia } \\ \text { Indiana } & \text { USA }\end{array}$


(Página deixada propositadamente em branco) 
Série

Investigação

Coimbra

Imprensa da Universidade

2000 